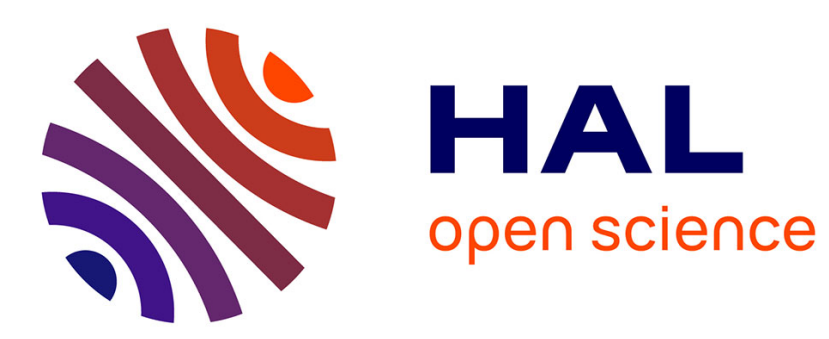

\title{
Réflexions sur l'avenir du système politique chinois Jean-Luc Domenach
}

\section{To cite this version:}

Jean-Luc Domenach. Réflexions sur l'avenir du système politique chinois. Revue Tiers Monde, 1996, 37 (147), pp.713-724. 10.3406/tiers.1996.5066 . hal-01010108

\section{HAL Id: hal-01010108 \\ https://hal-sciencespo.archives-ouvertes.fr/hal-01010108}

Submitted on 19 Jun 2014

HAL is a multi-disciplinary open access archive for the deposit and dissemination of scientific research documents, whether they are published or not. The documents may come from teaching and research institutions in France or abroad, or from public or private research centers.
L'archive ouverte pluridisciplinaire HAL, est destinée au dépôt et à la diffusion de documents scientifiques de niveau recherche, publiés ou non, émanant des établissements d'enseignement et de recherche français ou étrangers, des laboratoires publics ou privés. 


\section{Réflexions sur l'avenir du système politique chinois}

In: Tiers-Monde. 1996, tome 37 n¹47. La Chine après Deng (sous la direction de Claude Aubert, Jean-Pierre Cabestan et Françoise Lemoine) pp. 713-724.

Citer ce document / Cite this document :

Domenach Jean-Luc. Réflexions sur l'avenir du système politique chinois. In: Tiers-Monde. 1996, tome 37 n¹47. La Chine après Deng (sous la direction de Claude Aubert, Jean-Pierre Cabestan et Françoise Lemoine) pp. 713-724.

doi : 10.3406/tiers.1996.5066

http://www.persee.fr/web/revues/home/prescript/article/tiers_0040-7356_1996_num_37_147_5066 


\title{
RÉFLEXIONS SUR L'AVENIR DU SYSTĖME POLITIQUE CHINOIS
}

\author{
par Jean-Luc DOMENACH*
}

On distingue classiquement la notion de régime politique, qui désigne la manière dont sont organisés les pouvoirs publics, et celle de système politique "qui inclut non seulement l'organisation constitutionnelle des gouvernants, mais aussi d'autres acteurs et d'autres processus tels que, par exemple, le régime des partis, les libertés publiques et les médias, les mécanismes de socialisation politique des citoyens, etc. ${ }^{1}$. Dans les pays communistes, l'énorme puissance du Parti et de ses dirigeants s'étend bien au-delà de l'étroite sphère des institutions. C'est donc du système politique chinois qu'il va être surtout question au cours de cet article.

Celui-ci ne vise pas à dresser un tableau détaillé de ce système ni à recenser de façon exhaustive les courants et les forces politiques qui l'habitent. Les quelques pages qui suivent ont seulement pour objet de poser des jalons qui permettent une meilleure compréhension de l'environnement politique d'une Chine en pleine mutation. Mutations liées à l'essor économique, mutations aussi du personnel dirigeant en ces temps de succession. Ce sont donc de simples réflexions qui sont ici présentées, dont nous espérons qu'elles ne seront pas démenties par une actualité mouvante, dominée par les incertitudes liées à la fin de l'ère Deng Xiaoping.

\section{UNE CRISE DE SUCCESSION COMPLEXE}

Dans quelle direction ce système peut-il évoluer après la mort de son chef actuel ? La question est complexe parce que la disparition de Deng Xiaoping déclenchera inévitablement un processus d'événements nouveaux et difficiles à prévoir.

\footnotetext{
* Fondation nationale des sciences politiques, Paris.

1. Guy Hermet, Bertrand Badie, Pierre Birnbaum, Philippe Braud, Dictionnaire de la science politique et des institutions politiques, Armand Colin, 1994, p. 234-235.
} 
L'une des rares règles empiriques de l'histoire des pays communistes est que les successions y suscitent des crises politiques généralement plus décisives que tout autre événement économique, social ou diplomatique. Cette règle a été observée en Chine populaire puisque la disparition de Mao Zedong en 1976 a provoqué une crise certes différente de celles marquées par les terribles tragédies du Grand bond en avant et de la Révolution culturelle, mais en définitive plus décisive quant au tournant politique, aux mutations socio-économiques qui en ont résulté.

Comme toutes les règles, cependant, elle n'est pas absolue, ainsi que le montrent plusieurs expériences des autres systèmes communistes : par exemple, l'effondrement final de l'URSs en dehors d'une crise de succession, et la disparition des "démocraties populaires" d'Europe centrale sous l'effet d'un événement extérieur (la chute du mur de Berlin). En outre, les crises de succession ne produisent pas forcément des enchaînements imprévisibles: ce que Khrouchtchev avait tenté, puis Deng Xiaoping réalisé, un petit nombre de leurs collègues l'avaient prévu, et beaucoup de citoyens soviétiques et de Chinois l'avaient espéré. Leur programme constituait en effet la seule solution de survie du pouvoir communiste. En revanche, les experts étrangers, eux, ont rarement su deviner à temps où allaient les événements.

Le deuxième facteur de complexité est que, depuis cinquante ans et en particulier depuis l'arrivée au pouvoir de Deng Xiaoping, l'évolution du système politique chinois a fait que le nombre des partenaires d'une éventuelle crise de succession a augmenté et qu'ils se sont diversifiés. Aujourd'hui, la politique chinoise n'est plus seulement l'effet du jeu classique entre les appareils du Parti et la société. Il faut aussi prendre en compte l'influence des intérêts économiques plus ou moins privés, celle de certaines bureaucraties locales particulièrement fortes, enfin l'influence des étrangers : grandes puissances, pays voisins, Chinois d'outremer, sociétés multinationales. Il faut surtout peser le rôle de ces différents acteurs dans une crise de succession, c'est-à-dire dans un processus de sélection du nouveau chef et de consolidation (ou d'affaiblissement) de son pouvoir.

UN ITINÉRAIRE POLITIQUE ORIGINAL

Autre difficulté : l'évolution future se greffera sur un itinéraire politique déjà très original, depuis 1949, qu'il faut analyser rapidement.

Pour la première période, celle de Mao Zedong, l'analyse est facilitée par l'accumulation des travaux, par l'apaisement des passions et par le 
recul presque "historique " dont nous disposons. On parvient ainsi à un certain nombre d'hypothèses fortes qui annulent ou nuancent les affirmations plus ou moins polémiques qui avaient été autrefois proférées.

Rappelons tout d'abord que les formules toutes faites de la propagande chinoise ont été contredites par la grande masse des témoignages et des études ultérieures. Le système communiste, en Chine comme ailleurs, n'était pas un système populaire, il ne fonctionnait pas dans une fusion permanente entre le pouvoir et la population. C'était au contraire un système reposant sur des mécanismes de domination et de transformation volontariste.

La question est évidemment de savoir quel modèle d'explication peut le mieux rendre compte de la réalité d'un tel système. Jean Pierre Cabestan a proposé une grille d'analyse des différents modèles tour à tour utilisés, et qu'il est maintenant possible d'évaluer à la lumière des événements politiques chinois ${ }^{1}$. A notre sens, deux approches n'ont guère résisté à l'épreuve du temps : l'approche culturaliste (sauf à l'utiliser pour mettre en évidence des continuités socialement essentielles mais politiquement secondaires) et l'approche développementaliste (qui a eu sans doute plus de poids au cours de la période de modernisation suivante). Les autres approches paraissent, elles, plus compatibles avec les faits connus: c'est d'une part la théorie du totalitarisme, avec les nuances qui lui ont été apportées, et ce sont d'autre part ce que JeanPierre Cabestan appelle "les théories organisationnelles». C'est en les combinant plus ou moins explicitement que l'historiographie politique de la Chine populaire progresse actuellement.

Pour notre part, nous utilisons, depuis une dizaine d'années, une approche qui combine différentes hypothèses. La première est que c'est la théorie du totalitarisme qui rend encore le mieux compte de la nature fondamentale du système communiste chinois, à condition cependant d'y joindre d'autres facteurs majeurs : la culture politique complexe héritée de l'histoire impériale, l'élan nationaliste déclenché par l'agression occidentale et l'immensité des tâches de développement économique. Certes, du fait de ces facteurs spécifiques, les différences avec les autres systèmes communistes sont très importantes. Mais ces différences peuvent être mises en évidence à l'intérieur même du cadre d'analyse totalitaire constitué par Hannah Arendt et défini, entre autres, par Juan Linz $^{2}$. Il est clair, par exemple, que le système chinois a réservé une importance plus grande que le soviétique à la mobilisation idéologique

1. Jean-Pierre Cabestan, Le système politique de la Chine populaire, PUF, 1994, p. 34-39.

2. Juan Linz, Totalitarian and Authoritarian Regimes, in W. Polsby et E. Greenstein (ed.), Handbook of Political Science, Harvard University Press, 1975. 
et morale ainsi qu'au quadrillage spatial de la population. Mais, par leur architecture, les deux systèmes sont foncièrement analogues : ambition démiurgique, rôle du chef, monopoles idéologique, politique, répressif et économique, contrôle social et culturel, hostilité foncière aux démocraties et plus globalement au monde extérieur, tout y est.

Ce dispositif, cependant, est vivant, il ne cesse de se modifier, notamment par le jeu des différents échelons du pouvoir et par celui des réactions contrastées, variables et souvent ambiguës de la population. Le totalitarisme n'arrête pas l'histoire, il la structure et la nourrit ${ }^{1}$. Dans le cas chinois, comme dans le cas soviétique, cette histoire a été une histoire extrême et accidentée en ce qu'elle a produit des "pics" de violence totalitaire et des "plateaux " d'institutionnalisation. Du vivant même de Mao Zedong, des érosions sont donc apparues qui ont conduit le système totalitaire à la marge d'un autre monde : au début des années soixante et plus nettement dans la première partie des années soixantedix, l'impératif de la domination cède de plus en plus le pas aux nécessités du développement et de la coexistence internationale.

Pour comprendre ces évolutions, les approches "organisationnelles » développées aux États-Unis sont très utiles parce qu'elles éclairent, dans des recherches précises, cet autre moteur du développement du système politique chinois qui réside dans le dispositif spécifique de son organisation interne, avec ses fonctions, ses rouages, ses échelons, dans le contexte d'un immense pays rural. La particularité des travaux français est toutefois de combiner ce facteur "organisationnel » avec la prise en compte des réactions sociales. Nous nous sommes par exemple attaché à montrer comment, dans une province chinoise, il existait durant les années 1958-1960 une micro-histoire politique spécifique répondant non seulement aux disputes des factions pékinoises, mais à une histoire politique locale et à une crise sociale concrète ${ }^{2}$.

La combinaison de ces différentes approches permet par exemple de mieux comprendre la crise de succession ouverte par la mort de Mao en septembre 1976. L'approche totalitaire rend compte à la fois de l'immensité du problème ouvert par la disparition du démiurge et du fait que sa solution ne pouvait être trouvée, à moins d'un basculement dans une autre histoire, qu'à l'intérieur du PCC. L'approche "organisationnelle modifiée " éclaire le déroulement politico-social de la crise factionnelle qui part de Pékin, se développe dans les provinces puis revient à

1. Jean-Luc Domenach, La Chine ou les tribulations du totalitarisme, in Madeleine Grawitz et Jean Leca, Trailé de science politique, PUF, 1985, t. II, p. 238-267.

2. Jean-Luc Domenach, Aux origines du grand bond en avant, le cas d'une province chinoise, 1956-1958, Éditions de l'EHESS et Presses de la FNSP, 1982. On se référera aussi à l'histoire de la crise de succession de Mao Zedong publiée par Wojtek Zafanolli, Le président clairvoyant contre la veuve du Timonier. Payot, 1981. 
Pékin et trouve sa conclusion dans l'hiver 1978. Ces seules analyses structurelles, statiques, cependant, ne suffisent pas pour éclairer le sens dans lequel la crise a concrètement évolué. Une approche plus historique et plus sociale, telle que celle évoquée plus haut, devient là nécessaire. L'irruption du mécontentement populaire, la division profonde de l'encadrement politique ont favorisé l'irrésistible montée des partisans de Deng Xiaoping. Ils s'expliquent principalement par le fait que le « pic totalitaire » représenté par la Révolution culturelle avait déjà érodé la discipline sociale et le monolithisme idéologico-politique du Parti.

Dans cette succession, dans ce tournant décisif de l'histoire récente de la Chine populaire, un facteur très important ne relève toutefois d'aucun modèle spécifique, mais d'un aléa historique difficilement prévisible : c'est le facteur Deng Xiaoping. Le succès de Deng Xiaoping en décembre 1978 a dépendu de qualités qui n'étaient pas garanties d'avance malgré une carrière déjà longue et riche: sa santé physique (il avait déjà 74 ans...), son habileté manœuvrière et surtout son aptitude à produire une synthèse idéologique qui, dans le monde communiste au moins, n'avait pas de précédent, l'idée d'un «communisme modernisateur $»$.

UN SYSTÉme POLITIQUE ENIGMATIQUe

Comment définir le système politique ainsi re-fondé par Deng Xiaoping, tel qu'il s'est développé jusqu'en 1996 ? La réponse à cette question est aujourd'hui bien difficile, tant ce système paraît insaisissable, voire énigmatique. Cette réponse devrait en principe nous aider à comprendre la crise de succession qui s'annonce. Mais c'est bien plutôt cette crise de succession qui, dans une large mesure, dira la vérité sur la nature du système politique denguiste.

La première question qui se pose est évidemment celle de l'ampleur du changement imprimé en 1978. Jean-Pierre Cabestan remarque que, dans un texte daté de 1985, nous n'avons pas hésité à qualifier encore de totalitaire un régime communiste en pleine "démaoïsation » ${ }^{2}$. En effet, si elle insistait sur les «tribulations» du totalitarisme en Chine, notre analyse estimait que les monopoles essentiels (de l'idéologie, de la politique, de la violence) sur lesquels reposait le système politique chinois avaient survécu, tout en perdant de leur virulence. Avions-nous tort à l'époque et fallait-il déjà parler de " post-totalitarisme », voire d' " autoritarisme » 
pour définir le système denguiste? La question reste ouverte. Il serait trop facile d'arguer de la conclusion meurtrière de la crise du printemps 1989 pour montrer que le système n'avait pas foncièrement changé. En effet, la répression a été, malgré tout, moins terrible qu'elle ne l'aurait probablement été vingt ans plus tôt. Cette crise, ensuite, a été influencée par des données sociales et politiques nouvelles. Enfin, le système politique chinois lui-même avait déjà été transformé en profondeur par les effets des réformes économiques introduites par Deng Xiaoping.

Depuis, l'évolution du système a été relancée par les décisions que Deng Xiaoping a imposées en 1992, rendant encore plus insaisissable toute définition qui puisse lui être appliquée. D'un côté, cette relance a été l'effet de ce que l'on pourrait appeler un nouvel "événement totalitaire ": aux prises avec un blocage conservateur qu'il a lui-même créé (au lendemain du massacre de juin 1989), le chef sort des règles du régime et impose à la fois une purge (en l'occurrence limitée) et de nouvelles décisions. Mais celles-ci accentuent le compromis modernisateur de 1978 en promouvant une sorte de "communisme capitaliste». L'expression est certes trompeuse si elle prétend désigner la mise en place d'un "vrai » système capitaliste, avec un "vrai » marché et une "vraie " intégration aux échanges mondiaux (pour autant que ces expressions aient un sens). Mais elle désigne une évolution politique réelle que résume bien l'expression de "socialisme du marché »: le communisme ne se donne plus seulement pour priorité la modernisation économique, il passe publiquement un compromis avec le marché mondial, c'est-àdire avec une réalité extérieure qu'il intériorise en partie.

Incontestablement, nous sommes donc en présence d'un phénomène de transition, c'est-à-dire d'un processus qui combine de l'ancien et du nouveau. Ce processus n'est comparable en rien à celui qui a conduit les communismes d'Europe centrale et orientale à un effondrement subit. En effet, le pouvoir central reste pour l'essentiel monolithique, la société demeure à la fois cellularisée et dans l'ensemble contrôlée, et l'intégration au marché mondial est limitée de nombreuses façons. Ce phénomène est totalement original dans l'histoire communiste (sauf à prendre en compte le Vietnam, que la Chine a inspiré). On ne pourrait lui trouver d'équivalent, dans le monde capitaliste, que dans les transitions qu'ont connues les pays autoritaires d'Asie, dont, pourtant, les pouvoirs politiques étaient infiniment moins étendus.

L'ancien, c'est en effet la survie, au centre du système, de mécanismes de nature totalitaire : rôle du chef, grands monopoles politiques, répression policière. On peut certes noter que ces mécanismes sont partiellement décomposés, voire dégénérés. Le chef n'est plus guère, comme le Mao des dernières années, qu'une momie ridée s'exprimant par des 
borborygmes que la cour interprète aussitôt. L'appareil d'encadrement, gangrené par les luttes des factions et par la corruption, est à la fois moins et plus fragmenté qu'en 1976: moins fragmenté parce que les clivages n'ont plus guère de contenu politique, plus fragmenté dans la mesure où les fractures se développent en suivant des intérêts géographiques qu'il sera très difficile de réduire. La répression, enfin, est devenue à la fois moins meurtrière et plus aléatoire, sauf bien entendu lorsqu'elle frappe une dissidence politique de toute façon réduite.

Malgré cette dégénérescence, les mécanismes totalitaires perdurent au sommet du système politique. Ils expliquent que la politique chinoise demeure fondamentalement, comme à l'époque de Mao, un phénomène de cour. C'est entre les Dieux de l'Olympe chinois que se mijotent les grandes disputes et les petites querelles dont la prose du Quotidien du Peuple fournit ensuite une obscure traduction idéologique. Les Dieux n'ont certes plus le même pouvoir qu'autrefois sur les hommes et même sur les choses, mais rien ne se fait sans leur consentement, et ils ont encore leurs foucades. La Chine, par exemple, n'a plus guère de politique d'aménagement du territoire. Mais le vieux Chen Yun avait approuvé le projet pharaonique du Barrage des Trois Gorges, sur le Yang Tsé, auquel le premier ministre Li Peng a attaché son nom: malgré les doutes des scientifiques chinois et les réserves des organismes internationaux, ce projet est donc en voie de réalisation. En politique étrangère, les idées fixes de Jupiter s'ajoutent à l'inévitable complexité du jeu chinois pour aiguiser l'attitude de Pékin en direction de Taiwan: Deng Xiaoping a décidé, ni plus, ni moins, qu'il ne mourra qu'après avoir ramené dans le giron de la patrie non seulement Hong-kong mais encore l'île dissidente. Les mois à venir diront les conséquences d'une telle obsession.

Dans le même temps, et c'est le fait original et paradoxal de la période présente, on assiste à la renaissance et à l'extension d'une Chine peu ou pas concernée par les jeux de la politique totalitaire. L'innombrable paysannerie est en large part libérée de l'embrigadement politique d'autrefois. Elle subit les règlements centraux et les exactions des petits cadres, mais il existe aujourd'hui des possibilités de ruser et surtout de s'enrichir. L'encadrement local est encore autoritaire, mais déjà affairiste. Les situations varient et offrent des aspects contradictoires. Dans les villes, le pouvoir du Parti est en un sens plus fort que dans les campagnes, car le quadrillage est plus aisé. Inversement, c'est là que les mutations économiques et sociales sont les plus importantes, et que la toute-puissance de l'argent opère ses pires ravages. Le milieu citadin reste certes très polymorphe. Il n'est pas unifié par une idéologie nouvelle que porterait une "nouvelle bourgeoisie" en ascension. S'il com- 
porte encore une masse de peureux et de prudents, sa relation avec le pouvoir est cependant devenue complexe et évolutive, comme on l'a bien vu au printemps 1989 . Fondamentalement favorable à la modernisation économique et à la stabilité politique, il juge les autorités à leurs actes, non à leurs intentions. La même raison fait sans doute la surpuissance et l'impuissance du pouvoir politique : le fait qu'il ne rencontre guère plus d'adhésion que d'opposition.

Ces mutations sociales sont l'effet d'une évolution économique qui a fait apparaître de nouveaux acteurs "quasi politiques». Il y a d'abord les investisseurs étrangers, et surtout chinois d'outre-mer, qui possèdent leurs clients locaux. Ensuite les milieux d'affaires proprement chinois, liés au pouvoir politique mais professionnellement intéressés à l'ouverture et à la libéralisation économique. Il y a aussi les pouvoirs économiques provinciaux et locaux plus ou moins clairement identifiés. Enfin, dans la population, il faut noter le rôle possible de trois couches sociales dont le comportement en cas de crise politique prolongée est imprévisible: les paysans criblés d'impôts, les ouvriers des entreprises d'état menacés de chômage et les dizaines de millions de migrants.

Ainsi, pour reprendre notre distinction liminaire, on pourrait dire que la Chine actuelle possède encore un régime totalitaire, mais à la fois rétréci et affaibli, et que son système politique, lui, est devenu post-totalitaire, dans la mesure où le Parti dirigeant a totalement perdu le contrôle des esprits, et partiellement celui de l'économie et de la société.

UN AVENIR POLITIQUE INCERTAIN

Reste à savoir dans quelle direction la politique chinoise peut évoluer. L'énigme sera en partie levée à l'issue de la crise de succession qui s'annonce. Nous verrons alors dans quelle mesure l'aspiration populaire à la continuité et à la stabilité sera prise en compte, et si les milieux économiques chinois et étrangers pourront peser sur la cour et ses prétoriens. Nous saurons surtout où se situe désormais, dans un système en transition, la dynamique la plus puissante : dans le «bloc totalitaire primordial ", dans le camp du changement ou au contraire dans la rencontre - collision ou compromis - entre ces forces.

Aujourd'hui, seules sont possibles à notre sens deux remarques qui devraient engager les partenaires de la Chine à la prudence. La première est que, contrairement à une croyance répandue, la succession au sommet n'est nullement réglée. Le fait que Jiang Zemin serve actuellement de président délégué par le grand homme, et qu'il fasse tout pour gagner 
de l'autorité (notamment dans les milieux militaires et à l'étranger) ne signifie nullement qu'il y parviendra. A ce point de vue, l'histoire des successions en régime communiste ne fournit pas de probabilités claires, mais au contraire des cas très variés : absence de succession organisée (URSS, 1953), succession réussie (URSS, 1981 et 1985, et Corée du Nord, 1994); succession collective (Vietnam, 1969); succession échouée (Chine, 1976-1978). Les arguments généralement présentés en faveur du succès de Jiang Zemin sont importants, mais non décisifs. Il y a d'abord le fait, incontestable, qu'il a consolidé sa position depuis 1989 . Il y a aussi l'argument de la raison, selon lequel une telle solution permettrait, probablement, d'assurer un minimum de continuité, la transition autoritaire vers un "capitalisme chinois" se faisant tout en conservant la confiance des pays étrangers. Le premier argument est affaibli par le fait que les manœuvres en cours à Pékin et dans les provinces ne sont pas toutes favorables à Jiang, et surtout, par la possibilité pour les factions hostiles de se liguer contre lui. Le second argument est également contestable : il n'est pas certain, en effet, que tous les acteurs de la crise à venir seront sensibles à la "raison " (car l'attrait du pouvoir et de la richesse constitue en Chine aussi l'un des ressorts de la politique). Rien ne dit enfin que quiconque, autre que Deng Xiaoping, soit capable d'assurer la continuité d'une politique difficile et ambiguë.

La seconde remarque est que les mêmes facteurs qui ont assuré le dynamisme économique de la société chinoise depuis 1978 en font aussi, au cas où le contexte deviendrait incertain, une société politiquement dangereuse. Les forces productives ont été libérées: mais dans un désordre immense. Le communisme a disparu des esprits : mais rien ne l'a vraiment remplacé, si ce n'est le désir de l'argent. Le pouvoir reste trop fort pour que se développe une société civile; mais il est devenu trop faible pour interdire la montée des localismes. La modernisation économique a mis la Chine sur la voie du progrès, mais aussi fait apparaître des masses de mécontents. L'ouverture au monde a mis fin aux dangers extérieurs et à l'isolement technologique, mais elle a multiplié les occasions de fuite des cerveaux tandis que se développent par ailleurs des réactions xénophobes. Enfin, l'hypothèse d'une évolution « raisonnable " dépend largement des élites chinoises. Celles-ci sont techniquement bien mieux formées qu'autrefois, mais c'est aujourd'hui leur solidité morale qui fait question. Au total, donc, des maladresses ou des disputes publiques et durables entre dirigeants pourraient provoquer des événements que personne n'est en état d'imaginer.

Il faut manifester la même prudence à propos de l'avenir à moyen terme du système politique chinois. Une première inquiétude concerne l'évolution de la conjoncture internationale et régionale. Après avoir 
énormément pâti des menaces que lui adressaient ses voisins - d'abord le Japon militariste, puis l'URSs - la Chine a beaucoup bénéficié d'une série de grandes mutations successives : la montée d'une Asie capitaliste décidée à l'accueillir dans son sein; puis l'effondrement de l'URSs; ensuite, le nouveau climat international très affairiste d'après la guerre froide. Rien ne dit que l'environnement asiatique restera toujours aussi favorable, et que les produits chinois accéderont avec autant de facilité aux marchés occidentaux. Deuxième facteur de prudence: l'évolution économique. L'économie chinoise a brillamment profité, depuis quinze ans, d'un phénomène de "rattrapage " puis des effets d'une politique fondée sur des facteurs simples mais non éternels : une main-d'œuvre bon marché, des investissements en abondance, un ordre social relatif, des marchés extérieurs disponibles. Le rythme de la progression commence à ralentir, mais reste rapide. Se maintiendra-t-il si les facteurs de base viennent à se modifier? Enfin, la troisième inquiétude est d'ordre culturel. La Chine a toujours eu de brillants intellectuels, mais, par les temps qui courent, ceux-ci ont une fâcheuse tendance à émigrer ou à changer d'activité pour "plonger dans la mer" du business, comme on dit en Chine. Le résultat est que ce pays est pratiquement privé d'intelligentsia. Il a beaucoup d'idées (fort peu modestes) sur son passé, quelques-unes (rarement originales, car les censeurs veillent) sur son présent et pratiquement aucune sur son avenir.

Pour progresser dans la prévision, la seule solution consiste à partir d'un phénomène politique central - la crise du communisme - et à tenir compte des impératifs économiques et des influences de l'extérieur. La crise du communisme est bien le facteur dont tout le reste dépend. Il faut reconnaître tout de suite que, dans les conditions présentes, cette crise est au sens strict sans solution. En d'autres termes, reconnaître d'un côté que le communisme est privé de vitalité pour toutes sortes de raisons (désaffection populaire, corruption des élites, balance des forces internationales, etc.), et qu'il finira inévitablement par disparaître d'une façon ou d'une autre; mais aussi admettre que cette disparition n'est pas pour demain, compte tenu de la puissance installée du système (Parti, armée, police, etc.); noter enfin que le communisme laissera en Chine comme ailleurs des traces profondes. D'un autre côté, il faut également admettre qu'il n'existe pas de modèle politique apte à remplacer d'un coup le communisme défunt. En particulier, on l'a assez écrit, la démocratie pluraliste est actuellement inapplicable dans un pays socialement, psychologiquement et intellectuellement ravagé par plusieurs décennies de dictature et de misère, et qui au surplus n'en a guère la connaissance ni l'expérience. Pour autant, la démocratie constitue en Chine comme ailleurs une 
solution indépassable. Les élites chinoises y croient, bien souvent de façon naïve, et nombre de simples citoyens voient en elle le système des plus forts, qui s'étendra inévitablement à une Chine développée.

Nous venons de dire que sur le marché idéologique mondial, il n'existe pas de troisième modèle. Sur le marché régional, par contre, il en existe un : le modèle dit « asiatique » tel qu'il est actuellement pratiqué et prôné à Singapour et à Kuala Lumpur. Son attraction est réelle sur les milieux dirigeants chinois, parce qu'il pourrait assurer la transition économique sans modifier la répartition des pouvoirs. Elle s'exerce aussi sur certains cercles intellectuels - sans doute parce qu'elle satisfait à la fois leur prétention culturaliste et leurs intérêts professionnels. Ce modèle jouera donc un rôle. Mais son rayonnement rencontre de grands obstacles. Le premier est que le modèle " asiatique " n'a pas participé à la bataille historique binaire qui a occupé toute l'histoire du système politique chinois : communisme contre démocratie. En outre, il ne répond pas à l'aspiration universelle des sociétés post-totalitaires : l'aspiration à la liberté, à la jouissance individuelle, et donc au détachement des liens - il prétend au contraire les maintenir serrés. Enfin, il suppose une hostilité à l'Occident contraire à la fièvre majeure de la Chine d'aujourd'hui, qui est le désir d'Occident. Les citadins chinois rêvent de Californie, non de Singapour (où ils ne seraient d'ailleurs probablement pas accueillis). Le modèle "asiatique " ne devrait donc jouer qu'un rôle secondaire.

Du choc de deux avenirs impossibles ou improbables, ceux du communisme comme de la démocratie, que peut-il advenir ? Oui, que peut-il donc advenir quand de surcroît le politique se meut dans un espace surpeuplé, immense, très divers? Et surtout quand les camps ne sont pas clairement délimités mais s'interpénètrent et se mélangent ? A défaut d'issue tranchée, force est de considérer la possibilité de mélanges incertains et variables, de solutions intermédiaires et temporaires comme par exemple les modèles autoritaires et paternalistes. A moins que ne surgisse un modèle entièrement nouveau que par définition nous ne connaissons pas.

Une chose, toutefois, est certaine : la Chine a quitté l'ère des grandes ruptures, du rouge et du blanc, pour une ère de transitions, c'est-à-dire de mélanges et d'érosions, où les contraires se combinent et s'épuisent au lieu de s'affronter mortellement. En d'autres termes, la Chine est passée d'une ère de certitudes à une ère aléatoire.

Les aléas seront bien entendu historiques. Il est possible que la politique chinoise retombe dans une période d'hésitations, d'expérimentations, voire de troubles, comparable à celle qui avait suivi la chute de l'Empire en 1911. En faveur de cette hypothèse, il existe des arguments substantiels : l'incertitude des esprits, l'indiscipline sociale, les affrontements entre les factions, l'influence des pays étrangers. Mais la tragédie 
n'est ni certaine, ni même probable, car deux facteurs essentiels, qui font la différence avec les années vingt et trente en Chine, devraient limiter l'ampleur des désordres: une conjoncture extérieure dans l'ensemble favorable et une économie intérieure globalement en progrès très rapide, qui donc fait apparaître comme possible une issue collective aux drames et aux humiliations du passé. Pour cette raison, l'amplitude des aléas « historiques » pourrait être relativement réduite.

En revanche, les aléas "géographiques" peuvent être plus marqués. Nous ne trancherons pas ici, en quelques lignes, le cas d'école que constitue à cet égard la comparaison entre la Chine et l'URSS. Il existe des arguments forts en faveur de la thèse de l'éclatement de la Chine (la crise administrative et morale postcommuniste, les polarisations économiques, l'influence des voisinages). Il en existe d'autres, peut-être plus forts, qui vont à l'encontre de cette thèse (la masse du peuplement proprement chinois, les leviers conservés par Pékin, la faiblesse des minorités nationales, l'extrême prudence des pays voisins). Par-delà les hypothèses extrêmes, on peut imaginer une situation où, là aussi, les impossibilités pourraient se combiner en des formules différentes suivant les situations provinciales et locales. L'État central chinois survivrait, il conserverait les grandes responsabilités stratégiques, diplomatiques, économiques voire policières, et pour le reste négocierait son pouvoir avec les principales provinces et localités - le rapport des forces variant en fonction de la distance, de l'influence politique et de la puissance financière, voire militaire, des localités en question. La situation ainsi créée évoquerait celle de la Chine d'après l'Empire, avec deux différences probables (mais non certaines) : le (ou du) développement économique en plus, et la guerre civile en moins. Et, comme à cette époque, c'est sur les marges du pays, dans les zones de minorités nationales, qu'elle serait la moins contrôlée (Tibet, Xinjiang, Mongolie intérieure).

Ces quelques pages sur les avenirs possibles du système politique chinois, bien qu'elles tranchent par rapport aux prévisions généralement optimistes des partenaires commerciaux de la Chine, ne procèdent d'aucun a priori. Comme celles qui les ont naguère précédées, ces réflexions ont seulement pour intention d'éprouver une continuité interprétative entre les différentes phases historiques de la politique chinoise. De même que le totalitarisme n'épuisait ni n'arrêtait l'histoire chinoise, de même le posttotalitarisme politiquement dangereux mais économiquement triomphant dans lequel ce pays est engagé ne garantit nullement une issue claire. En Chine comme ailleurs, il n'y a pas de prédestination: l'histoire politique reste l'affaire des "forces profondes", mais aussi des circonstances, et des hommes. 